\title{
Dysfunction of neuronal calcium signaling in aging and disease
}

\author{
Ana M. M. Oliveira $\cdot$ Hilmar Bading $•$ Daniela Mauceri
}

Published online: 13 July 2014

(C) Springer-Verlag Berlin Heidelberg 2014

Calcium has been selected by evolution as a key regulator of cellular functions. Many physiological processes are dependent on intracellular calcium. This has required cells to develop efficient mechanisms for the tight control of calcium homeostasis. Basal cytosolic and nuclear calcium levels are low but they can increase dramatically within milliseconds after exposure to external stimuli. The way in which the cell responds to changes in calcium concentration depends on the magnitude, temporal and spatial resolution of the calcium transients. In the central nervous system, calcium signals have numerous functions. These include synaptic transmission, which is central to neuronal communication and several forms of synaptic plasticity, behavioral adaptions and gene transcription. Since many vital physiological processes depend on intracellular calcium, disturbances of calcium homeostasis can lead to cellular dysfunction and degeneration. In this special issue, a collection of review articles addresses the dysregulation of calcium homeostasis and associated proteins in aging and various pathological conditions of the nervous system. In addition, the reviews discuss new therapeutic approaches aimed at the normalization of calcium-dependent mechanisms.

Calcium plays a critical role in the regulation of neuronal excitability. This can be achieved directly, via the modulation of the activity of ion channels and indirectly, through the activation of signaling pathways. Therefore, unsurprisingly, calcium is viewed as an important factor in neuronal hyperexcitability, seizure generation and epileptogenesis. Steinlein (2014) describes the way that calcium-dependent signaling is involved in epilepsy. The author discusses both

A. M. M. Oliveira $(\square) \cdot$ H. Bading $\cdot$ D. Mauceri $(\square)$

Department of Neurobiology, Interdisciplinary Centre for

Neurosciences (IZN), University of Heidelberg, INF 364,

69120 Heidelberg, Germany

e-mail: oliveira@nbio.uni-heidelberg.de

e-mail: mauceri@nbio.uni-heidelberg.de genetic epilepsies, in which mutations in calcium channels have been detected and acquired epilepsy, which is the consequence of brain trauma, stroke, infection, or tumors. Insults such as brain trauma and stroke produce an intracellular calcium overload that can render neuronal networks vulnerable to seizures and even trigger cell death. The author acknowledges the fact that, even if the dissection of the calcium signaling pathways involved in epilepsy is a daunting task, it promises to uncover targets for the development of new antiepileptic drugs.

Schaefer et al. (2014) focus on the effects of the cerebral ischemia that is associated with various brain insults and on proteins involved in mitochondrial calcium homeostasis. Ischemia is defined as a decrease in blood and oxygen supply to the brain caused by constriction or obstruction of blood vessels. Oxygen deprivation disrupts oxidative phosphorylation and thus ATP synthesis. Under physiological conditions, calcium influx into mitochondria can stimulate mitochondrial ATP production in order to meet the energy demands of the cell. Schaefer et al. (2014) provide an overview of the current knowledge of the manner in which ischemia can disturb bioenergetics and possibly lead ultimately to cell death. Strategies supporting mitochondrial function and motility during periods of reduced blood and oxygen supply should confer neuroprotection under cerebral ischemia conditions.

Hagenston and Simonetti (2014) highlight the crucial role of calcium signaling and its homeostasis in the regulation of physiological and pathological pain. The authors provide a systematic and comprehensive description of the various sources of calcium entry into neuronal cells in chronic pain development. The authors summarize the well-established notion that alterations both in the expression and in the functioning of several calcium channels can be linked to mechanisms of peripheral and central sensitization. In addition, Hagenston and Simonetti (2014) discuss the 
importance of calcium sinks and extrusion processes in the mechanisms underlying pain sensitization. The authors also consider the molecular and cellular effects of calcium homeostasis dysregulation in the various forms of pathological pain: chronic inflammatory, neuropathic and diabetic neuropathy. Given the plethora of effects that calcium dysregulation has in pathological pain, including the regulation of both gene transcription and neuronal architecture, the design of novel therapeutic strategies targeting the mechanisms regulating calcium levels in pain states might be beneficial.

The importance of calcium homeostasis in brain function is also addressed in the review by Brawek and Garaschuk (2014) who concentrate on the dysregulation of calcium homeostasis in Alzheimer's disease (AD). In this review, the authors describe the alterations in calcium homeostasis that characterize the early features of $\mathrm{AD}$ and are thought to be a consequence of amyloid $\beta(A \beta)$ production. They describe several mechanisms by which $A \beta$ has been suggested to alter calcium regulation and the way that these alterations can result in an imbalance of diverse signaling pathways, seizure susceptibility and cognitive deficits, which are all hallmarks of AD. Moreover, the authors put forward the hypothesis that the dysregulation of neuronal calcium homeostasis in the $\mathrm{AD}$ brain also depends on the activation of inflammatory responses in glial cells and that the bidirectional interaction of the two factors represents a vicious cycle accelerating disease progression. Brawek and Garaschuk (2014) suggest that the targeting of intracellular calcium stores in order to modulate intracellular calcium levels might be an effective therapeutic strategy to combat disease progression. Indeed, the results of some studies point in this direction.

The review by Rush and Buisson (2014) further explores the topic of calcium homeostasis alterations as a consequence of $A \beta$ production in $\mathrm{AD}$. Their focus is on the role of the calcium-permeable N-methyl-D-aspartate (NMDA) receptors and on the opposing function these receptors can have on cell survival depending on their localization and subunit composition. The authors point out that a shift in the balance between the pathways mediated by synaptic NMDA receptors that facilitate non-amyloidogenic processing and the extrasynaptic NMDA receptors that promote $A \beta$ production can mediate altered synaptic plasticity and neurotoxicity. They describe mechanisms by which this balance can be perturbed as a consequence of $A \beta$ oligomer production. The importance of targeting the extrasynaptic NMDA receptors for AD amelioration is highlighted by the fact that memantine is the only NMDA receptor antagonist approved for $\mathrm{AD}$ treatment. Memantine preferentially inhibits extrasynaptic NMDA receptors.

Alteration of calcium homeostasis has also been linked to another neurodegenerative disorder, Parkinson's disease (PD). Calì et al. (2014) examine the connection between calcium signaling and PD. They review the possibility that the selective vulnerability typical of the dopaminergic neurons of the substantia nigra ( $\mathrm{SNc}$ ) can be partially ascribed to their autonomous activity sustained by calcium channels. SNc neurons indeed differ from most neurons of the central nervous system as they trigger action potentials even in the absence of inputs. Cali et al. (2014) focus on the calcium-buffering systems of dopaminergic neurons, which are altered in PD. The authors argue that autonomous activity promotes basal metabolic stress, which is, under normal conditions, kept under control by mitochondrial calcium buffering. During aging or after exposure to stressors, mitochondrial control systems fail and neurodegeneration takes place. Calì et al. (2014) discuss the various molecules that have been implicated in the dysfunction of calcium homeostasis in dopaminergic neurons.

Neurodegeneration attributable to the dysregulation of calcium homeostasis is further observed in certain inflammatory autoimmune conditions. Fairless et al. (2014) discuss the link between calcium signaling and multiple sclerosis (MS). They explain the way in which, during the course of MS and of its experimental animal model (experimental autoimmune encephalomyelitis), degeneration can be ascribed to an abnormal calcium load of the neuronal cells. The authors provide a comprehensive overview of the changes in the expression, subcellular localization and intrinsic properties of calcium channels and pumps. Fairless et al. (2014) report that inflammatory autoimmune disorders are accompanied by aberrations of calcium homeostasis not only in neurons but also in T cells and glial cells. They discuss the possibility of normalizing the function and localization of calcium channels as a novel therapeutic strategy.

Calcium channels also take center stage in the review by Berger and Bartsch (2014). Here, the authors discuss the importance of L-type voltage gated calcium channels in physiological and pathological brain function. Berger and Bartsch (2014) explain the functional role that these channels play in cognitive functions such as learning and memory, the formation of fear and the reward system. The authors summarize current knowledge concerning genetic alterations of these calcium channels in neuropsychiatric disorders, including Timothy syndrome, bipolar disorders (BPDs), major depression and schizophrenia. Like Calì et al. (2014), they also discuss the link between the selective vulnerability of the dopaminergic neurons of the SNc, autonomous activity and calcium channel aberrations. Finally, they offer perspectives on future therapies designed to modulate the functionality of the L-type voltage gated calcium channels.

Calcium signaling in psychiatric disorders is discussed in depth by Berridge (2014). This review introduces and explains the complex concepts of brain rhythms and the balance between excitatory and inhibitory (E-I) transmission. Berridge (2014) discusses the possible role of E-I imbalance in schizophrenia and BPD and puts forward the hypothesis that aberrations of the E-I balance can be ascribed to alterations of the calcium-dependent transcription of key signaling elements. 
The author argues that, in spite of their different behavioral patterns, schizophrenia and BPD might share similar cellular and molecular changes. In addition, Berridge (2014) describes the role of calcium signaling in the mechanisms of the action of lithium and valproate, the most commonly used drugs for the treatment of BPD. The author focuses not only on the inositol depletion hypothesis that he has postulated in the past but also on a novel hypothesis that is based on neurogenesis.

In recent years, the importance of glial cells in brain pathologies has become evident. Verkhratsky et al. (2014) provide a thorough overview of the various neuroglia cell types and their possible contribution to a range of disorders. Moreover, Verkhratsky et al. (2014) discuss the cellular and molecular changes of glial cells during normal aging and report glial dysfunctions in several neurological disorders such as inflammation, neurodegeneration, developmental failures and psychiatric disorders. The importance of glia cells in brain function is also highlighted in other reviews in this issue. Steinlein (2014) discusses alterations in calcium signaling in astrocytes in the context of epilepsy. These have been implicated in the initial imbalance between inhibitory and excitatory activities that functions as a starting point for seizure generation. Brawek and Garaschuk (2014) propose a network-wide theory for calcium dyshomeostasis in AD by interconnecting alterations in neuronal calcium signaling and inflammatory responses of glial cells that, in a feed-forward process, accelerate disease progression. Rush and Buisson (2014) point out that the astrocytic release of glutamate to the extrasynaptic space triggered by $\mathrm{A} \beta$ contributes to the activation of the extrasynaptic NMDA receptor, a major death-promoting event.

This Special Issue of Cell and Tissue Research is meant to introduce the reader to the importance of calcium signaling in nervous system function and dysfunction. The reviews address diverse pathophysiological aspects of cellular calcium signaling and discuss the source of calcium entry, intracellular calcium-buffering systems, cellular substrates, cascades of calcium signal propagation and calcium-mediated transcription. The reviews also highlight the importance of calcium signaling in the regulation of neuronal-glial networks, brain circuits and behavior. The contribution of calcium signaling and of its dysregulation in many neurological conditions is not unexpected. Therefore, therapeutic strategies aimed at restoring calcium homeostasis represent a promising new path toward the treatment of brain diseases.

We are grateful to our colleagues who have contributed to this Special Issue of Cell and Tissue Research. We also thank the Editor-in-Chief, Klaus Unsicker, for inviting us to be guest editors of the present Special Issue and his editorial assistant, Maite Menes, for her continuous and patient support.

\section{References}

Berger M, Bartsch D (2014) The role of L-type voltage gated calcium channels Cav1.2 and Cav1.3 in normal and pathological brain function. Cell Tissue Res. doi:10.1007/s00441-014-1936-3

Berridge MJ (2014) Calcium signalling and psychiatric disease: bipolar disorder and schizophrenia. Cell Tissue Res. doi:10.1007/s00441014-1806-Z

Brawek B, Garaschuk O (2014) Network-wide dysregulation of calcium homeostasis in Alzheimer's disease. Cell Tissue Res. doi:10.1007/ s00441-014-1798-8

Calì T, Ottolini D, Brini M (2014) Calcium signaling in Parkinson's disease. Cell Tissue Res. doi:10.1007/s00441-014-1866-0

Fairless R, Williams SK, Diem R (2014) Dysfunction of neuronal calcium signalling in neuroinflammation and-degeneration. Cell Tissue Res. doi:10.1007/s00441-013-1758-8

Hagenston AM, Simonetti M (2014) Neuronal calcium signaling in chronic pain. Cell Tissue Res. doi:10.1007/s00441-014-1942-5

Rush T, Buisson A (2014) Reciprocal disruption of neuronal signaling and $A \beta$ production mediated by extrasynaptic NMDA receptors: a downward spiral. Cell Tissue Res. doi:10.1007/s00441-013-1789-1

Schäfer MKE, Pfeiffer A, Jaeckel M, Pouya A, Dolga AM, Methner A (2014) Regulators of mitochondrial $\mathrm{Ca}^{2+}$ homeostasis in cerebral ischemia. Cell Tissue Res. doi:10.1007/s00441-014-1807-y

Steinlein OK (2014) Calcium signaling and epilepsy. Cell Tissue Res. doi:10.1007/s00441-014-1849-1

Verkhratsky A, Rodriguez JJ, Parpura V (2014) Neuroglia in ageing and disease. Cell Tissue Res. doi:10.1007/s00441-014-1814-Z 